\title{
COMPUTATIONAL PREDICTION OF THE SUPRAMOLECULAR
}

\section{SELF-ASSEMBLING PROPERTIES OF ORGANIC MOLECULES:}

\section{FLEXIBILITY $v s$ RIGIDITY}

\section{A PREPRINT}

\author{
Laura Le Bras* \\ Unité de Chimie Physique \\ Théorique et Structurale, \\ Chemistry Department, \\ Namur Institute of Structured Matter, \\ University of Namur, Belgium \\ laura.lebras@unamur.be
}

\author{
Yves L. Dory \\ Laboratoire de Synthèse Supramoléculaire, \\ Département de Chimie, \\ Institut de Pharmacologie, \\ Université de Sherbrooke, \\ Sherbrooke, Québec, Canada
}

\section{Benoît Champagne*}

Unité de Chimie Physique

Théorique et Structurale, Chemistry Department, Namur Institute of Structured Matter,

University of Namur, Belgium

benoit. champagne@unamur.be

March 26, 2021 


\begin{abstract}
Two families of organic molecules with different backbones have been considered. The first family, composed by a substituted central phenyl is considered as flexible. The second one, based on a macrolactam-like unit, is considered as rigid. They have however a common feature, three amide moieties (as substituents for the phenyl and within the cycle for the macrolactam-like molecule) that allow hydrogen bonding when molecules are stacked. In this study we propose a computational protocol to unravel the ability of the different families to self-assemble into organic nanotubes. Starting from the monomer and going towards larger assemblies like dimers, trimers, and pentamers we applied different theoretical protocols to rationalize the behavior of the different assemblies. Both structures and thermodynamics were investigated to give a complete picture of the process. Thanks to the combination of a quantum mechanics approach and molecular dynamics simulations along with the use of tailored tools (non covalent interaction visualization) and techniques (umbrella sampling), we have been able to differentiate the two families and highlight the best candidate for self-assembling purposes.
\end{abstract}

\title{
1 Introduction
}

Supramolecular chemistry is a key concept for many edifices or mechanisms that are essential for life [1]. So it is for some applications going from material sciences to medicine through information storage[2, 3, 4. 5, 6]. It has been defined by Lehn, one of its founders, as the chemistry of intermolecular bonds and interactions [7]. Supramolecular chemistry involves a wide variety of weak interactions of different strength. Metal-ligand interactions are the strongest one with an interaction energy of about $80 \mathrm{kcal}^{\mathrm{mol}}{ }^{-1}$. Ionic, ion-dipole and dipole-dipole interactions are slightly less stabilizing with an interaction energy ranging from 15 to $50 \mathrm{kcal} . \mathrm{mol}^{-1}$. Aromatic interactions, encompassing $\pi$ - $\pi, \pi$-cation and $\pi$-hydrogen bond, lead to a bounding of $3 \mathrm{kcal}^{\mathrm{mol}}{ }^{-1}$. Finally, hydrogen bonds and van der Waals forces are the less energetic ones with interactions energies of $1-10 \mathrm{kcal}_{\mathrm{mol}}^{-1}$ and lower than $1 \mathrm{kcal} \cdot \mathrm{mol}^{-1}$, respectively. As the previously mentioned interactions are non-covalent, it means that the interactions can be reversible and thus confer a kind of flexibility to the system. Nevertheless, increasing the number or combining weak interactions can lead to really stable assemblies and one can cite for example the structure of ice, DNA [8] or the synthetic supramolecular polymer, namely the nylon[9]. In both cases, hydrogen bonds (H-Bonds) are at the origin of the large stability of the supramolecular assemblies (SMA). The association and dissociation processes within large SMA is ensured by the inter- and intramolecular H-Bonds, the best example being the secondary and tertiary structures of proteins that are dictated by those weak interactions [10, 11, 12]. In particular, H-Bonds 
based on an amide pattern $(\mathrm{N}-\mathrm{H} \cdots \mathrm{O}=)$ are frequently found. The low to medium strength of this type of H-Bond, around $2 \mathrm{kcal} . \mathrm{mol}-1$, along with its flexibility, allow SMA to assemble and disassemble easily. One of the specificity of the amide-based H-Bonds is their directionality. It has been used to build SMA that are characterized by a stacking of the molecules leading to a one-dimensional columnar SMA [13, 14]. Many studies have reported SMA structures where the building block was a substituted benzene with various number of amide moieties for the formation of organogels or liquid crystals [15, 16, 17, 18]. The number and the orientation of the amide moieties have been shown to be crucial for the effectiveness of the formation of the stacking[13].

Based on the idea of an amide-substituted benzene for the formation of columnar stacking, we have designed four benzenetricarboxamide molecules (B4s family, Figure 1). Amide moieties are perpendicular to the plane of the benzene. A difference in the orientation of the three amide moieties was observed for B4s family, while two of them were oriented in the same direction, the last one was found antiparallel (see Figure S3 in ESI $\dagger$ ). The remaining positions of the central benzene were substituted by $-\mathrm{CH}_{3}(\mathbf{B 4}, \mathbf{B} 4 \mathbf{c}, \mathbf{B} 4 \mathbf{p})$ or $-\mathrm{I}(\mathbf{B} 4 \mathbf{p I})$ groups to maintain the amide moieties perpendicular to the plane of the molecule. In addition to the substitution of the central benzene, amides were also substituted by $-\mathrm{C}_{6} \mathrm{H}_{13}$ alkane chain (B4), $-\mathrm{C}_{6} \mathrm{H}_{11}$ cyclic group (B4c), and $\mathrm{CH}_{2}-\mathrm{Ph}$ moiety for both B4p and B4pI. A different backbone, based on a macrolactam-like unit, has been considered (B9s family, Figure 1). It also encompassed three amide moieties that are conjugated to an alcene to avoid their rotation. Two substitution patterns have been added, a $-\mathrm{CH}_{3}$ for $\mathbf{B 9 m}$ and a $-\mathrm{CH}_{2} \mathrm{OCOCH}_{3}$ for B9. The global idea is to compare those two apparently C3-symmetric backbones for an efficient formation of columnar stacking involving amide-based H-Bonds.

To address this challenge we propose a computational approach. Recent studies have already shown that a fully theoretical approach, based on molecular dynamics simulations and quantum chemical calculations, was able to unravel hydrogen bond interactions between organic molecules within supramolecular assemblies[19]. Hence, the multiscale approach that will be developed in this study will address for the different small organic molecules (See Figure 1) their self-assembling property and the stability of the possible columnar stackings, considered as organic nanotubes. The multiscale approach will involve calculations based on quantum mechanics but also classical molecular dynamics simulations combined with tailored tools to characterize the H-Bonds. Umbrella sampling is particularly relevant for the analysis of supramolecular interactions as it allows the calculation of a binding energy through the derivation of a potential of mean force [20, 21, 22, 23]. Non-covalent interactions (NCI) visualization is also a valuable tool that can illustrate the underlying weak interactions such as hydrogen bonds or van der Waals interactions within SMA [24, 25]. 


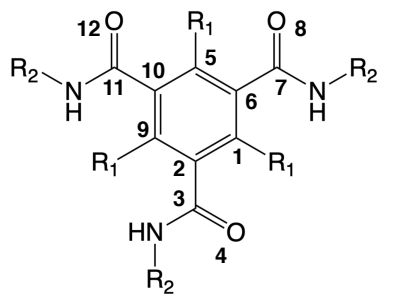

$$
\begin{aligned}
& \text { B4 : } \mathrm{R}_{1}=-\mathrm{CH}_{3} ; \mathrm{R}_{2}=-\mathrm{C}_{6} \mathrm{H}_{13} \\
& \text { B4c }: \mathrm{R}_{1}=-\mathrm{CH}_{3} ; \mathrm{R}_{2}=-\mathrm{C}_{6} \mathrm{H}_{11} \\
& \text { B4p : } \mathrm{R}_{1}=-\mathrm{CH}_{3} ; \mathrm{R}_{2}=-\mathrm{CH}_{2} \mathrm{Ph} \\
& \text { B4pl : } \mathrm{R}_{1}=-\mathrm{I} ; \mathrm{R}_{2}=-\mathrm{CH}_{2} \mathrm{PH}
\end{aligned}
$$

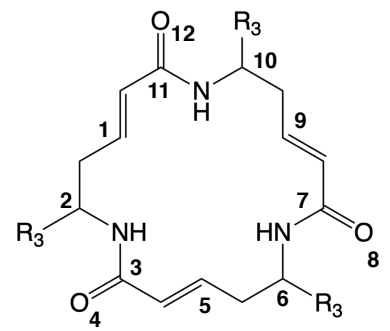

B9 : $\mathrm{R}_{3}=-\mathrm{CH}_{3}$

B9m : $\mathrm{R}_{3}=-\mathrm{CH}_{2} \mathrm{OCOCH}_{3}$

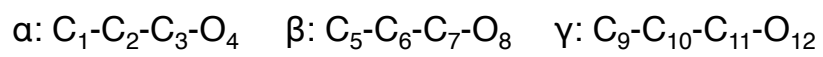

Figure 1: Representation of the structures of the four molecules of the B4s family, B4, B4c, B4p and B4pI and of the two molecules of the B9s familly, B9 and $\mathbf{B 9 m}$. The three important dihedral angles are also defined.

\section{Computational Details}

\subsection{Quantum Mechanics}

\subsubsection{Single point calculations}

All the calculations have been performed using Gaussian16 package [26] within the density functional theory (DFT) framework. We used the $\omega$ B97X-D range-separated hybrid exchange correlation functional (XCF) combined with the 6-311+G(d,p) atomic basis set [27]. This XCF is known as one of the most efficient to consider structures and energies of assemblies involving hydrogen bond interactions[28]. The solvent (water) was modeled using an implicit solvation model, namely the polarizable continuum model (PCM) [29]. In order to describe large assemblies, we relied on a QM/QM' ONIOM approach. Within this model it is necessary to define 2 subsystems. The model system, composed of the central phenyl and the amide groups for $\mathbf{B} 4 \mathbf{s}$ and the macrolactam-like unit for B9s, will be described at both the high level of theory $(\omega \mathrm{B} 97 \mathrm{X}-\mathrm{D} / 6-311+\mathrm{G}(\mathrm{d}, \mathrm{p}))$ and the low level $(\mathrm{HF} / 3-21 \mathrm{G}(\mathrm{d}))$ of theory. The real system, encompassing the model system and the lateral groups of both families, will only be treated at the low level of theory. A charge embedding framework was also added to the hybrid QM/QM' ONIOM scheme [30]. 


\subsubsection{Non-covalent interactions}

It has been possible to visualize non-covalent interactions (NCI) through the use of NCIPLOT code[31]. NCI analysis gives rise to an index that is based on the calculated electronic density and its reduced gradient, represented as a two-dimensional plot. For a given system, there will be a drastic change in the reduced density gradient (RDG) between the atoms that are interacting, leading to density critical points. The latter can be represented on the molecular structure as an isosurface to indicate the region where a weak interaction is occuring. Nevertheless, both attractive (H-Bonds, van der Waals) and repulsive (steric repulsion) interactions can be spotted thanks to this index. By looking at the second derivative of the density and to the sign of its eigenvalue it is possible to distinguish attractive and repulsive interactions. Hence the density and the sign of the eigenvalue of the density second derivative give information about the strength and the type of interaction respectively and one can visualize them via isosurfaces. The electronic density used to compute the NCI index is the one calculated at the same level of theory as the one presented in Section 2.1.1.

\subsection{Molecular Dynamics}

\subsubsection{Classical Molecular Dynamics}

Molecular dynamics (MD) simulations were run with the generalized AMBER force field (GAFF) [32] within GROMACS 2018.3 package[33]. As GAFF is not directly implemented in GROMACS, we used acpypi script [34, 35, 36] to convert the files from AMBER to GROMACS formalism. For each molecule, the atomic charges were derived following the parametrization procedure in GAFF, that is to say using HF/6-31G(d) RESP charges. The validity of the force field was checked by comparing the structures obtained after an optimization process in vacuum with GAFF and with DFT at the $\omega \mathrm{B} 97 \mathrm{X}-\mathrm{D} / 6-311+\mathrm{G}(\mathrm{d}, \mathrm{p})$ level. Results are provided in the Supporting Information section.

During the simulations, the system is composed by $n$ organic molecules ( $n$ being equal to 1, 2, 3, 5, and 10 and the organic molecules being either from B4s or B9s families). The general philosophy of our molecular dynamics simulations is represented schematically on Figure 2 The size of the simulation box and the number of water molecules depend on the system under investigation. All those information for each system under investigation are gathered in Supporting Information.

To describe the electrostatic interactions, periodic boundary conditions were imposed along with a cut-off of $10 \AA$ and the use of the Particle Mesh Ewald (PME) method [37, 38]. Following a steepest descents minimization, each system was equilibrated in two steps. For the first step, a simulation in the canonical ensemble (NVT) during 100 ps was carried out. The temperature was set to $310 \mathrm{~K}$ using the Berendsen weak 

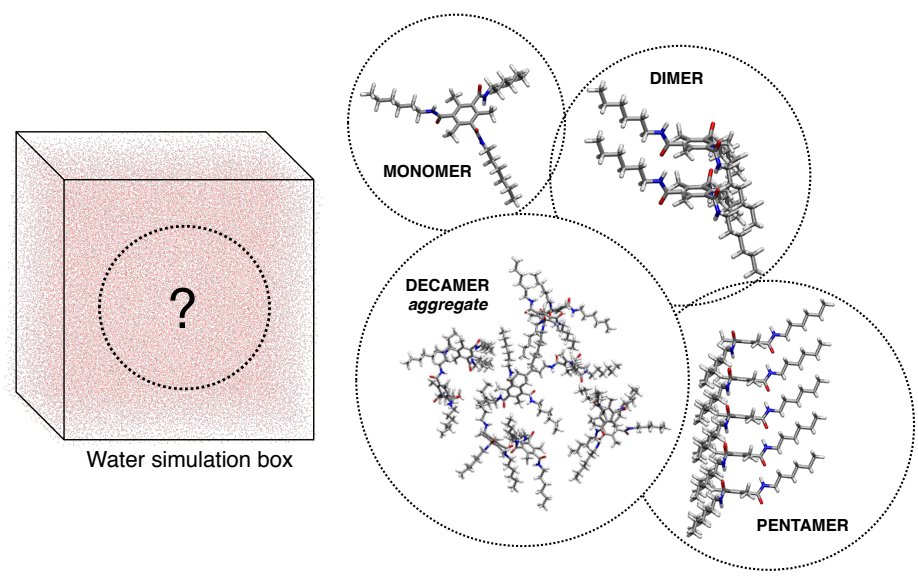

Figure 2: Schematic representation of our strategy for molecular dynamics simulations. Within a simulation box full of water molecules we will successively consider either monomers, dimers, pentamers or aggregates composed of ten monomers.

coupling method[39]. Organic molecules and solvent were coupled to separate temperature coupling baths. For the following second step, simulation under constant pressure (NPT) was performed. To maintain an isotropic pressure of 1 bar, we relied on the previously mentioned weak-coupling Berendsen method. The production phase was then carried out in the same NPT ensemble. Temperature was controlled thanks to the Nosé-Hoover thermostat while the isotropic character of the pressure was maintained via the Parinello-Rahman barostat. Combining this thermostat and barostat ensures the presence of a true NPT ensemble. Simulation time, if not explicitly precised, was set to $10 \mathrm{~ns}$.

\subsubsection{Umbrella sampling}

Within the umbrella sampling (US) approach we have considered dimers, trimers and pentamers. The systems were placed in a rectangular box that can allow a pulling simulation (e.g a simulation box that is too small will lead to an interaction with the periodic images). In particular, the $z$ length had to be large enough in order to satisfy the minimum image convention. The solvent molecules (water), were described through the TIP3P model. The first step consisted in an NPT equilibration of $100 \mathrm{ps,} \mathrm{as} \mathrm{it} \mathrm{was} \mathrm{described} \mathrm{above.} \mathrm{For} \mathrm{the} \mathrm{proper}$ pulling simulation, restraints were applied to one of the monomers for dimers, to a dimer for trimers and to a tetramer in the case of the pentamer. The molecules that were restrained were thus considered as immobile references. The molecule that was not restrained was then pulled away from the immobile one, along the $z$-axis over $500 \mathrm{ps}$ at a rate of $0.1 \mathrm{~nm} \cdot \mathrm{ps}^{-1}$ with a spring constant of $250 \mathrm{~kJ} \cdot \mathrm{mol}^{-1} \mathrm{~nm}^{-2}$. The final COM (center of mass) distance between the two considered assemblies that was obtained was $4 \mathrm{~nm}$. Snapshots were extracted from this pulling simulation in order to be as many starting points for the different umbrella sampling windows. For COM distances under $1 \mathrm{~nm}$, a separation of $0.05 \mathrm{~nm}$ was considered between each window and 


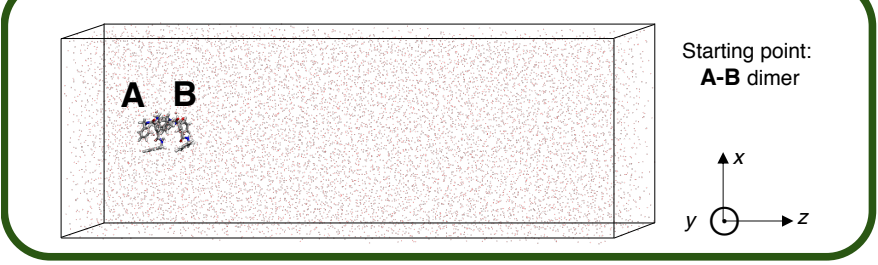

(b) Pulling simulation

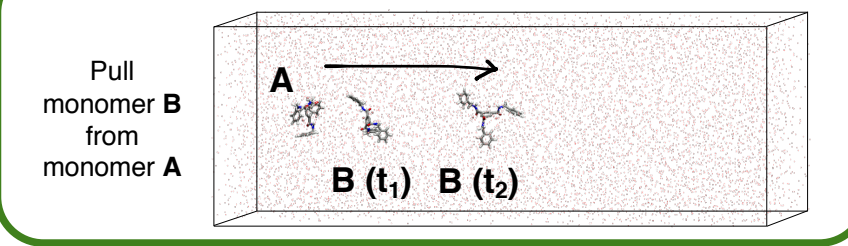

(c) Umbrella sampling simulations

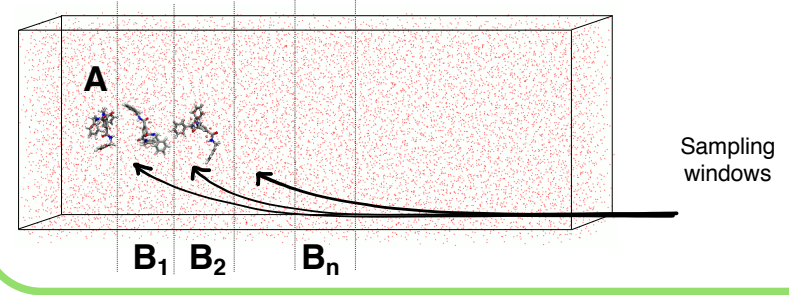

(d) Probability

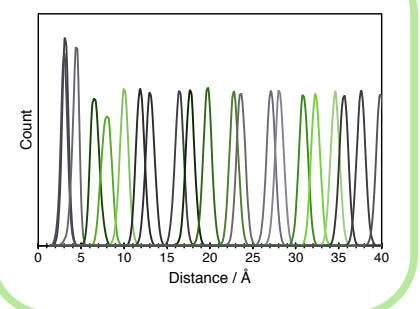

(e) PMF profile

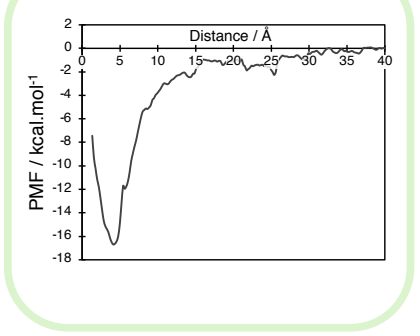

Figure 3: Schematic representation of the umbrella sampling approach. With (a) the starting configuration for a dimer. The dimer is positioned voluntarily on one side of the simulation box and the $z$-axis is voluntarily set to a large value to allow the next pulling step. (b) Pulling simulation. B is pulled away from A at a constant rate. (c) Along the pulling simulations, different configurations with different $A-B$ distances $\left(A-B_{1}, A-B_{2}\right)$ are extracted in order to be as many starting configuration for different sampling window simulations that will be centered on the A-B distance. (d) For each sampling window, we count the number of times that the A-B distance is equal to each value. (e) A Potential of Mean Force can be reconstructed and can be associated to the free binding energy, $\Delta \mathrm{G}_{\text {bind }}$.

then, for COM distances above $1 \mathrm{~nm}$ and up to $2.5 \mathrm{~nm}$, the spacing between the windows was $0.1 \mathrm{~nm}$. An example of the corresponding histogram is provided in Supporting Information. It allows a smoother and more accurate description of the interaction at small COM distances. This approach leads to around 25 windows. 
For each window, a $10 \mathrm{~ns}$ simulation was performed, resulting in a total simulation time of $250 \mathrm{~ns}$ for the US approach, for each assembly of each molecule. The analysis of the results was done using the Weight Histogram Analyzis Method (WHAM), implemented in the GROMACS 2018.3 package[40]. A schematic representation of the umbrella sampling approach is provided in Figure 3

\section{Results and discussion}

\subsection{Monomers}

Key structural parameters (see Figure 1 for their definitions) have been selected to study the different monomers. For both B4s and B9s families, three dihedral angles were defined $(\alpha, \beta$, and $\gamma)$, illustrating the relative position of the amide groups with respect to the plane of the molecule. We followed their evolution along the $10 \mathrm{~ns}$ MD simulations. The average values are reported in Table 1 .

Table 1: Average values of the selected dihedral angles (in degrees) defined on Figure 1 along with the RMSD value (in $\AA$ ) for each of the molecules. Standard deviations are provided in parentheses.

\begin{tabular}{r|cccc}
\hline & $\alpha$ & $\beta$ & $\gamma$ & RMSD \\
\hline B4 & $-90(13)$ & $91(14)$ & $88(13)$ & $2.33(0.48)$ \\
B4c & $-90(13)$ & $89(14)$ & $89(13)$ & $2.08(0.48)$ \\
B4p & $-89(13)$ & $90(13)$ & $90(13)$ & $3.07(0.56)$ \\
B4pI & $-88(14)$ & $90(12)$ & $89(13)$ & $3.12(0.51)$ \\
B9 & $95(7)$ & $95(6)$ & $96(8)$ & $0.44(0.12)$ \\
B9m & $83(7)$ & $83(7)$ & $84(8)$ & $0.80(0.13)$ \\
\hline
\end{tabular}

For the B4s family, as previously mentioned, all the amide moieties are not oriented in the same direction. As illustrated by $\alpha$ that is negative, there is one amide moiety that is antiparallel to the two other ones for $\mathbf{B} 4 \mathbf{s}$. This feature will thus induce a difference for the stacking behavior of the next steps. For the B9s family, all the amide groups are oriented in the same direction. During the MD simulations, all the dihedral angle values are close to ideal value of $90^{\circ}$ and no complete rotation of the amide moieties was observed. It indicates that all the amide moieties are and stay perpendicular to the plan of the molecule. Considering the standard deviation associated to those values, $\mathbf{B 9}$ and $\mathbf{B 9 m}$ show a smaller flexibility with deviations around $7^{\circ}$ while this value increases up to $14^{\circ}$ for $\mathbf{B} 4 \mathbf{s}$ family. To confirm this hypothesis, we computed the root mean square deviation (RMSD) of the different structures along the simulation, the reference structure being the ideal one with perfectly perpendicular amide groups. One has to notice that for the RMSD calculation, the hydrogen atoms were not considered. The results are gathered in Figure 4 and Table 1 


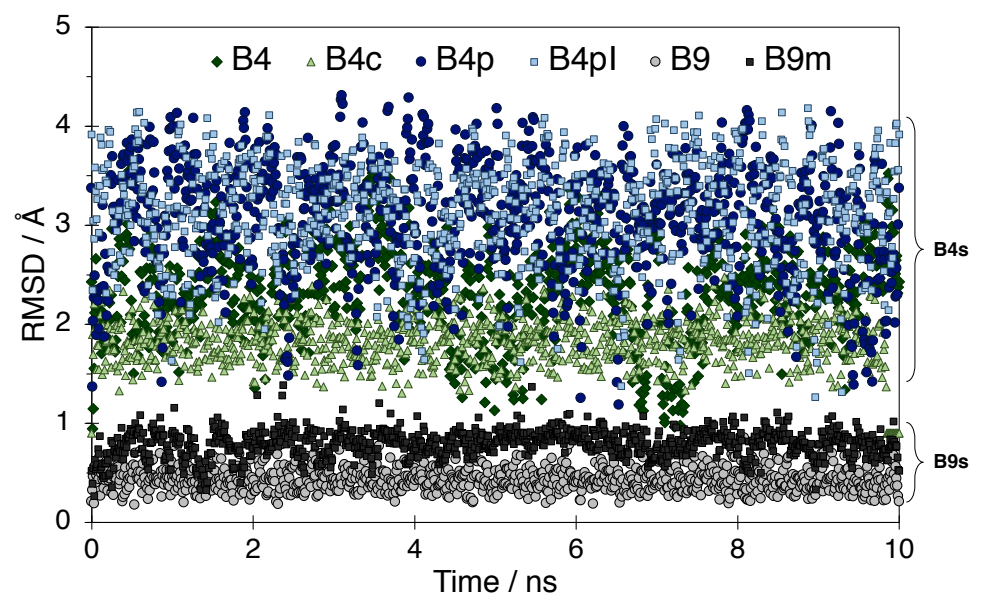

Figure 4: Evolution of the RMSD values, in $\AA$, for the considered molecules along the simulation time. Hydrogen atoms were not considered for the calculation of the RMSD.

The two families can be clearly distinguished. RMSD values are lower than $1 \AA$ for $\mathbf{B 9}$ and $\mathbf{B 9 m}$ while they are systematically larger than $1.5 \AA$ for $\mathbf{B} 4 \mathbf{s}$ family. It indicates that during the simulations, B4s molecules are more flexible than the B9s. Due to the inclusion of the amide groups within the macrolactam-like backbone of B9s, there is almost no degree of freedom for the molecule, as illustrated by the low average RMSD value for this family ( 0.44 and $0.80 \AA$ for $\mathbf{B 9}$ and $\mathbf{B 9 m}$ respectively). Fluctuations are also small with deviations equal to 0.12 and $0.13 \AA$ for $\mathbf{B 9}$ and $\mathbf{B 9 m}$ respectively. On the other hand, for $\mathbf{B} 4 \mathbf{s}$ molecules, the average RMSD is ranging from $2.08 \AA$ to $3.12 \AA$ for $\mathbf{B 4} \mathbf{c}$ and $\mathbf{B 4} \mathbf{p I}$ respectively, indicating quite large structural modifications during the simulation. Standard deviations confirm this trend with values around $0.50 \AA$ for the four molecules of B4s family. Studying the monomers of the different families has highlighted the fact that one family can be considered as rigid (B9s) while the other one appears to be more flexible $(\mathbf{B} 4 \mathbf{s})$. This feature may have an impact if one wants to build larger supramolecular assemblies as it will ease, or not, the formation of hydrogen bonds. The next sections are dedicated to the comparison of the two families for the formation and the stabilities of different SMA.

\subsection{Dimers}

\subsubsection{Qualitative approach}

To study the possible self-assembling behavior of the different molecules, we decided to consider the smallest and thus simplest supramolecular entity, namely a dimer. The interaction of the two monomers is ensured by the amide moieties. Indeed it is possible to form a hydrogen bond between the $\mathrm{N}-\mathrm{H}$ part of the amide of one molecule and the $\mathrm{C}=\mathrm{O}$ bond of another amide. As previously observed during the study of the monomers, the 
amide groups are perpendicular to the plan of the molecule and it is thus possible to stack monomers via a network of three hydrogen bonds. The dimers we built are represented on Figure 5
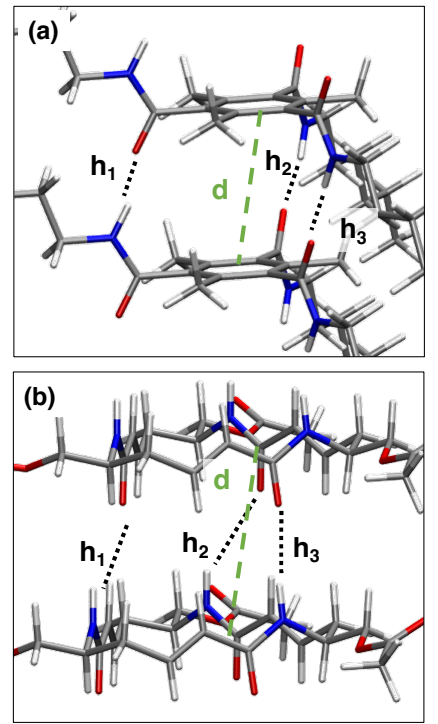

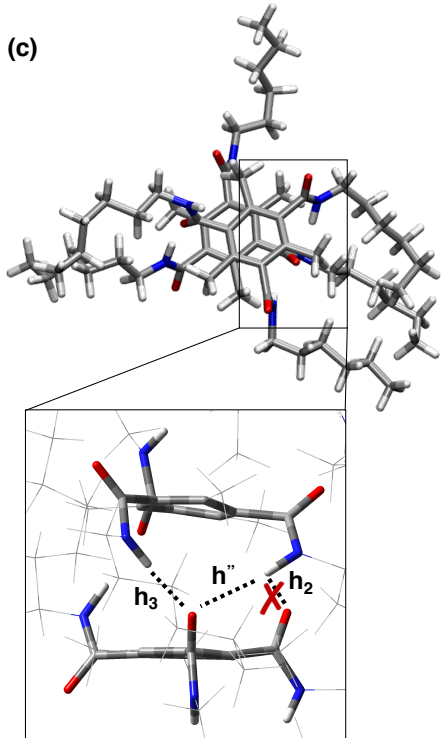

Figure 5: Representation of the stacking of the dimers for the (a) B4s and (b) B9s families. Key distances are also identified for both stackings. (c) Representation of a particular stacking observed for B4s and definition of a new hydrogen bond, h'.

MD simulations were performed for each dimer. Three new key structural parameters were introduced, namely $\mathrm{h}_{1}, \mathrm{~h}_{2}$, and $\mathrm{h}_{3}$, which illustrate the three hydrogen bonds and $\mathrm{d}$, that illustrates the distance between the centroids of the central phenyl for B4s and of the macrolactam-like unit for B9s. The average values for each dimer are gathered in Table 2.

By looking at the average value of $\mathrm{d}$ along the MD simulations it is already possible to have a qualitative idea of the stability of the different assemblies. If all the dimers involving monomers from the B4s family are stable, it is not the case for the B9s one. Indeed, the $\mathrm{d}$ values that are reported in Table 2 illustrate the fact that the two monomers involved within a dimer are remaining close to each other. If we go deeper in the analysis, we observe that the average value of $\mathrm{d}$, ranging from $3.77 \AA$ to $4.23 \AA$ for $\mathbf{B} 4 \mathbf{s}$, is close to the crystalline characteristic value (4.8 $\mathrm{A}$ ) for such a stacking involving amide-based H-Bonds [41, 42, 43]. One has to notice that the standard deviations presented in Table 2 around $0.2-0.3 \AA$, illustrate the fact that the dimers are not "broken" along the simulation. The same conclusion can be drawn for B9m with an average value of $3.82 \AA$. As the dimer involving $\mathbf{B 9}$ monomers were not stable, it was not relevant to measure $\mathrm{d}$ along the simulation. One has to notice that if $\mathrm{h}_{1}, \mathrm{~h}_{2}$, and $\mathrm{h}_{3}$ are equivalent for B9s family, they are not for B4s dimers. Because of the asymmetry in the orientation of the amide moieties, only $h_{2}$ and $h_{3}$ are equivalent while $h_{1}$ is unique. 
Table 2: Average values, in $\AA$, for key distances of the different dimers. The mean deviations are also reported. The corresponding simulation time, in ns, during which the averages have been calculated is also provided in parentheses.

\begin{tabular}{|c|c|c|c|c|c|c|}
\hline & B4 & B4c & B4p & B4pI & B9 & B9m \\
\hline$\overline{\mathrm{d}}$ & $\begin{array}{c}4.23 \pm 0.30 \\
(10)\end{array}$ & $\begin{array}{c}4.12 \pm 0.23 \\
(10)\end{array}$ & $\begin{array}{c}4.11 \pm 0.30 \\
(10)\end{array}$ & $\begin{array}{c}3.77 \pm 0.27 \\
(10)\end{array}$ & $\begin{array}{l}- \\
-\end{array}$ & $\begin{array}{c}3.82 \pm 0.23 \\
(10)\end{array}$ \\
\hline $\mathrm{h}_{1}$ & $\begin{array}{c}2.05 \pm 0.23^{a} \\
(10)\end{array}$ & $\begin{array}{c}2.05 \pm 0.34 \\
(10)\end{array}$ & $\begin{array}{c}1.99 \pm 0.24 \\
(10)\end{array}$ & $\begin{array}{c}2.01 \pm 0.20 \\
(10)\end{array}$ & $\begin{array}{l}- \\
-\end{array}$ & $\begin{array}{c}2.03 \pm 0.22 \\
(10)\end{array}$ \\
\hline $\mathrm{h}_{2}$ & $\begin{array}{c}2.49 \pm 0.66^{a} \\
(8.4)\end{array}$ & $\begin{array}{c}2.81 \pm 0.61 \\
(7.2)\end{array}$ & $\begin{array}{c}2.49 \pm 0.58 \\
(8.9)\end{array}$ & $\begin{array}{c}2.59 \pm 0.71 \\
(3.0)\end{array}$ & $\begin{array}{l}- \\
-\end{array}$ & $\begin{array}{c}2.01 \pm 0.18 \\
(10)\end{array}$ \\
\hline $\mathrm{h}_{3}$ & $\begin{array}{c}2.54 \pm 0.64^{a} \\
(3.1)\end{array}$ & $\begin{array}{c}2.08 \pm 0.35 \\
(1.1)\end{array}$ & $\begin{array}{c}2.62 \pm 0.63 \\
(3.2)\end{array}$ & $\begin{array}{c}2.64 \pm 0.67 \\
(6.4)\end{array}$ & $\begin{array}{l}- \\
-\end{array}$ & $\begin{array}{c}2.02 \pm 0.21 \\
(10)\end{array}$ \\
\hline h' & $\begin{array}{c}2.44 \pm 0.60^{a} \\
(1.2)\end{array}$ & $\begin{array}{c}3.17 \pm 0.66 \\
(0.5)\end{array}$ & $\begin{array}{c}2.24 \pm 0.45 \\
(9.3)\end{array}$ & $\begin{array}{c}2.26 \pm 0.49 \\
(6.3)\end{array}$ & $\begin{array}{l}- \\
-\end{array}$ & $\begin{array}{l}- \\
-\end{array}$ \\
\hline h" & $\begin{array}{c}2.39 \pm 0.53^{a} \\
(7.7)\end{array}$ & $\begin{array}{c}2.20 \pm 0.50 \\
(8.8)\end{array}$ & - & $\begin{array}{c}2.36 \pm 0.61 \\
(2.3)\end{array}$ & $\begin{array}{l}- \\
-\end{array}$ & $\begin{array}{l}- \\
-\end{array}$ \\
\hline
\end{tabular}

${ }^{a}$ For the calculation of the average value and the associated standard deviation, only the areas highlighted on Figure 6 are considered. The same procedure is used for the other molecules, averages are made when the bonds are effective during the corresponding time.

Looking at those different values gives insights into the interactions involved in the stability of the dimers. We have represented on Figure 6 the evolution of $h_{i}$ distances for $\mathbf{B} 4$.

(a)

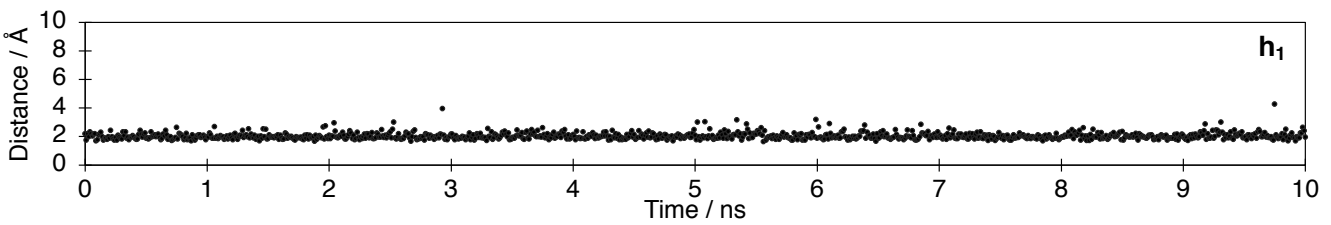

(b)

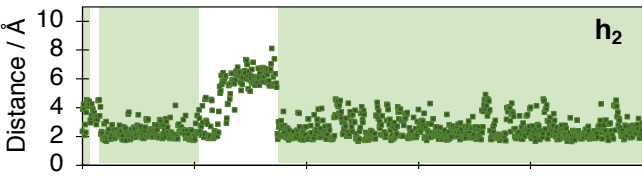

(c)

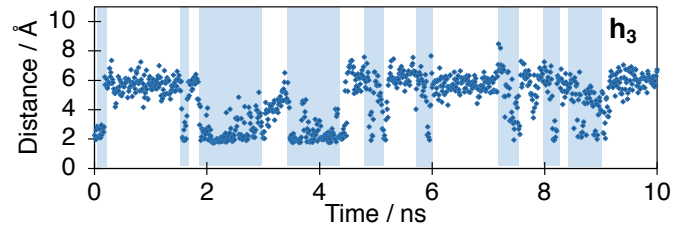

(d)

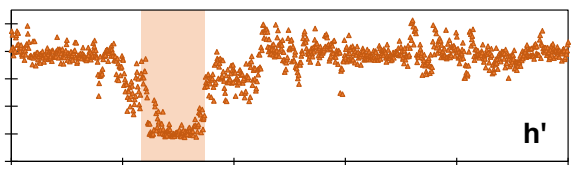

(e)

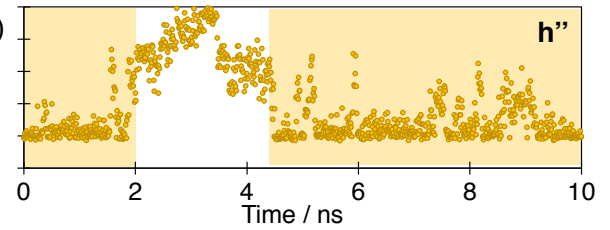

(f)

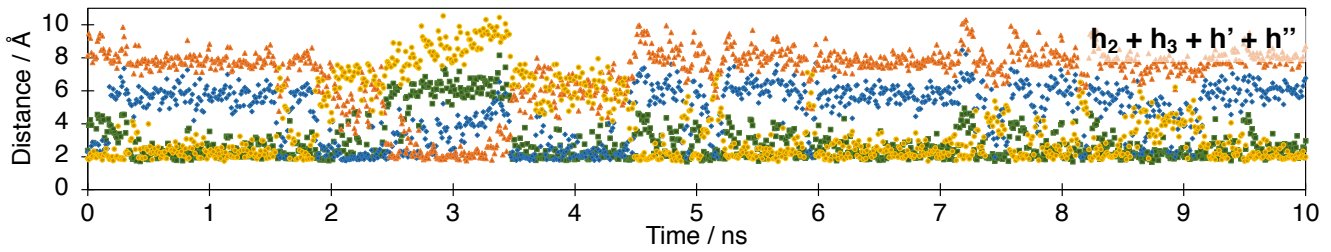

Figure 6: Evolution for B4 dimer of the distances (a) $h_{1}$, (b) $h_{2}$, (c) $h_{3}$, (d) h', (e) h". On (f), he, h $, h^{\prime}, h^{\prime}$ distances have been gathered. All the distances are in $\AA$. 
It is clear from Figure 6 a) that the hydrogen bond $\mathrm{h}_{1}$ is always effective during the $10 \mathrm{~ns}$ simulation. Moreover, with an average value of $1.99 \pm 0.24 \AA$ this hydrogen bond is within the range (1.5-3.5 $\mathrm{\AA}$ ) of a hydrogen bond with a medium strength (4 to $15 \mathrm{kcal} . \mathrm{mol}-1$ ). The same conclusion can be drawn for all the dimers of the $\mathbf{B} 4 \mathbf{s}$ family. The analysis is quite trickier for $h_{2}$ and $h_{3}$. As represented on Figure $6 \mathrm{p}$ and $c$, those H-Bonds are not always effective. The area that are colored represent the moment where $h_{2}$ and $h_{3}$ distances are within the 1.5-3.5 A range. The time corresponding to the colored area are given in Table 2 If $\mathrm{h}_{2}$ is effective during almost the $10 \mathrm{~ns}$ simulation ( $8.4 \mathrm{~ns}$ ), it is not the case for $\mathrm{h}_{3}$, that is only effective transiently for $3.1 \mathrm{~ns}$. Nevertheless, the dimer of $\mathbf{B} \mathbf{4}$ is stable and the stacking is maintained during all the simulation (d distance gives this information). By looking closer to the structure along the trajectory it has been possible to highlight a particular structure (Figure 5 5 c)). During the simulation the two monomers do not stay aligned. There is a tilt of one monomer with respect to the other one, inducing an hybrid hydrogen bonding. The keto part of one amide points between two amino moieties of two other amides of the other monomer. One expected H-bond is thus presents $\left(h_{2}\right.$ or $\left.h_{3}\right)$, even if it is longer than a perfectly aligned H-bond, while the other one is new. We have thus introduced two new H-bonds, namely h' and h" (Figure 5/c)). Their evolutions are represented on Figure 6 and e. One can observe that either h' or h" is effective during the simulation. Their average values along with their effective time are gathered in Table 2 It is possible to say that the H-Bonds complement each other. When $h_{2}$ is not effective, one can observe that $h$ ' is. When $h_{3}$ is not effective, $h$ " is taking over. We decided to combine those four H-Bonds on the same graphics (Figure 6 e e)). It appears that there is always at least one but most of the time two hydrogen bonds ( $h_{2}, h_{3}, h$ ', or $h$ ") that is present within the dimer, ensuring its stability along with $h_{1}$. The Boltzmann population ratio for the normal (with $h_{1}, h_{2}$ and $h_{3}$ ) and the hybrid (with $h_{1}$ and $h_{2} / h$ " or $h_{3} / h$ ') dimer (see Figure S4 in ESI $\dagger$ ) is always around 40/60 for all the dimers of the B4s family. Indicating that the hybrid dimer is more favorable than the normal one. For the other dimers of the B4s family, the same behavior is observed with a compensation of $h_{2}$ and $h_{3}$ by $h$ ' and $h$ " to ensure the global stability of the dimers. For $\mathbf{B 9 m}$ dimer, $\mathrm{h}_{1}, \mathrm{~h}_{2}$, and $\mathrm{h}_{3}$ are effective during all the trajectory but no hybrid H-Bond can be observed as there is no sufficient flexibility for the amide moieties.

To summarize the findings about dimers, we have been able to highlight (i) the (non-)stability of B4s family and B9m (B9), (ii) the effectiveness of the hydrogen bond network to build supramolecular assemblies and (iii) an hybrid H-bond pattern allowed by the flexibility and the possibility for $\mathbf{B} 4 \mathbf{s}$ dimers to orient their amide moieties. 


\subsubsection{Quantitative approach: Umbrella Sampling}

To go further in assessing the stability of the different dimers, we have undertaken US simulations. It allows us to retrieve the binding free energy, $\Delta \mathrm{G}$, along a reaction coordinate, $x$, that represents the preferential direction for the stacking pattern. Using around 25 sampling windows along this axis, one can construct a one-dimensional potential of mean force (PMF) profile for each system under study, leading to a binding energy, $\mathrm{E}_{\text {bind }}$. US simulations for larger assemblies, namely a trimer and a pentamer, were also performed. For each PMF profile, the minimum energy is associated to a particular distance, $\mathrm{d}_{\text {com }}$, that can be roughly compared to the $\mathrm{d}$ distance discussed in the previous section as it is the distance between the center of masses of the different assemblies. All those values, $\mathrm{E}_{\text {bind }}$ and $\mathrm{d}_{\text {com }}$, for each system and for each molecule, are gathered in Table 3 For the following discussion, the comparison and evolution of $\mathrm{E}_{\text {bind }}$ will be made on the absolute value.

Table 3: Computed binding free energy, $\mathrm{E}_{\text {bind }}$, in $\mathrm{kcal}^{\mathrm{mol}}{ }^{-1}$ for different assemblies and the corresponding optimal interaction distance, $\mathrm{d}_{\text {com }}$, in $\AA$.

\begin{tabular}{r|cccccc}
\hline & & Dimer & & \multicolumn{2}{c}{ Trimer } & \multicolumn{2}{c}{ Pentamer } \\
& $\mathrm{E}_{\text {bind }}$ & $\mathrm{d}_{\text {com }}$ & $\mathrm{E}_{\text {bind }}$ & $\mathrm{d}_{\text {com }}$ & $\mathrm{E}_{\text {bind }}$ & $\mathrm{d}_{\text {com }}$ \\
\hline B4 & 17 & 4.2 & 21 & 4.3 & 15 & 4.3 \\
B4c & 24 & 4.2 & 17 & 4.0 & 12 & 4.1 \\
B4p & 12 & 4.2 & 12 & 3.9 & 8 & 4.1 \\
B4pI & 21 & 3.6 & 23 & 3.9 & 22 & 3.8 \\
B9 & 7 & 3.8 & 9 & 3.8 & 7 & 4.2 \\
B9m & 12 & 4.2 & 6 & 4.0 & 4 & 4.1 \\
\hline
\end{tabular}

The binding energy that are obtained for the B4s dimers are globally higher than the one for the B9s. They are equal to $17,24,12$, and $21{\mathrm{~kJ} . \mathrm{mol}^{-1}}^{-1}$ for $\mathbf{B 4}, \mathbf{B} 4 \mathbf{c}, \mathbf{B} 4 \mathbf{p}$, and $\mathbf{B} 4 \mathbf{p I}$ respectively while they are equal to 7

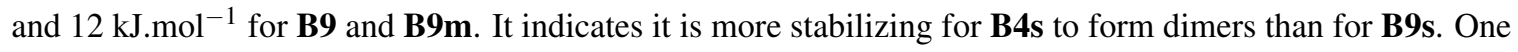
can notice that the US $d_{\text {com }}$ values are consistent with the MD average values, with a distance around $4.0 \AA$ between the two monomers, corresponding to a classic stacking distance for this kind of molecules.

The question that motivated the new simulations involving trimers and pentamers is the following: How is the binding energy evolving when the supramolecular assembly is getting larger? More specifically, is it getting harder to add a monomer to a dimer, a tetramer? The answer to this question will help us to understand the self-supramolecular assembling behavior of B4s and B9s into larger assemblies. The same umbrella sampling approach presented before was used to study first the interaction within a trimer. Two subsystems were considered, a dimer and a monomer and we were looking for the binding energy of the monomer with the dimer. For the pentamer, the binding energy is computed for the interaction of a tetramer and a monomer. The PMF profile for trimers and pentamers, for each molecule, is provided on Figure 7 . For B4s family, the 
A PREPRINT - MARCH 26, 2021
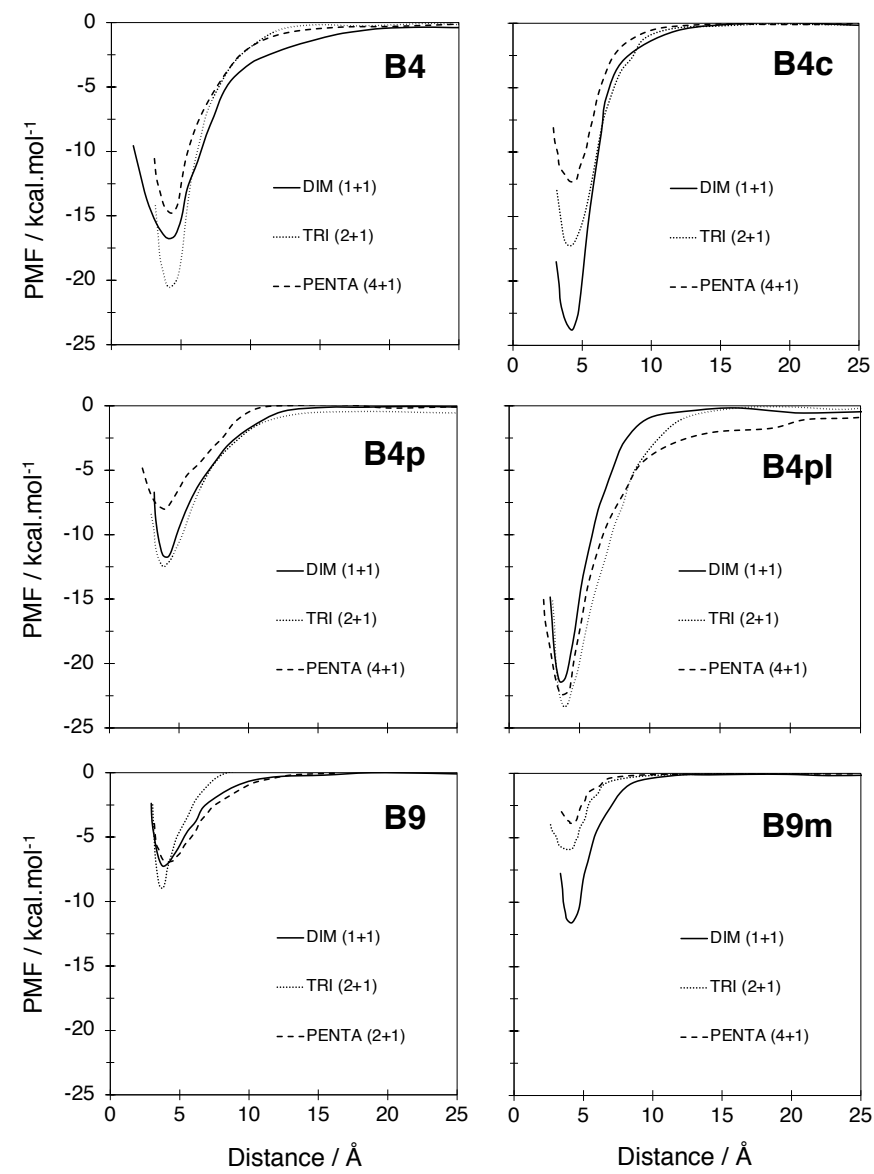

Figure 7: Potential of mean force (PMF) curves for each of the molecules in different configurations: dimer (bold line), trimer (dotted line) and pentamer (dashed line).

three possible different behavior are observed, a slight increase of $\mathrm{E}_{\text {bind }}$ for B4 $(+4)$ and $\mathbf{B 4} \mathbf{p I}(+2)$, a decrease for B4c (-7) and no evolution for B4. One can nevertheless notice that the formation of trimers still remain favorable in all the cases. For $\mathbf{B 9}$ and $\mathbf{B 9} \mathbf{m}, \mathrm{E}_{\text {bind }}$ is slightly increasing (+2) and decreasing (-6) respectively. In conclusion, adding a monomer to an already formed dimer is more favorable for $\mathbf{B} 4 \mathbf{s}$ than for B9s. Trimers of B4s appear even more stable than dimers of B9s. Going further and considering pentamers for both families leads to a unique conclusion. The binding energy is always decreasing when compared to the energies obtained for dimers and trimers. Nevertheless, the values obtained for $\mathbf{B 4 s}\left(15,12,8\right.$, and $22 \mathrm{kcal}^{\mathrm{mol}}{ }^{-1}$ for B4, B4c, B4p, and B4pI respectvively) are still higher than the ones obtained for B9s ( 7 and $4 \mathrm{kcal}_{\mathrm{mol}}{ }^{-1}$ for $\mathbf{B 9}$ and B9m respectively), indicating that adding a monomer to a small oligomer of B9s family will be less favorable than for the B4s family. 


\subsection{Pentamers}

Classical molecular dynamics simulations involving pentamers have been performed for each of the molecules. Two behaviors were observed for the two families. For B9s, almost no H-bond interactions were maintained throughout the trajectory leading to a non-stability for both of the assemblies. Though, dimers were observed ponctually (see Figure S8 and S9 in ESI $\dagger$ ). The conclusions that were drawn in the previous sections are thus confirmed with (1) a low but still possible stability for dimers and (2) an unfavorable binding energy for systems involving a large number of monomers.

(a)

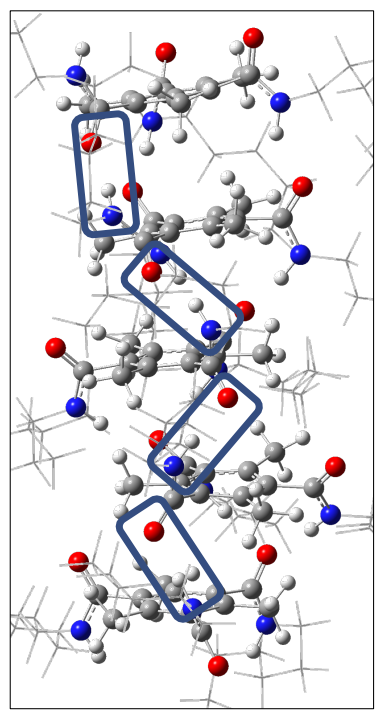

(b)

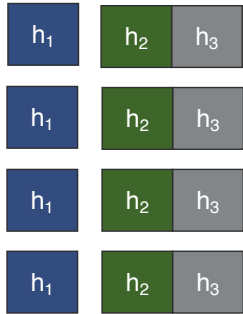

(c)

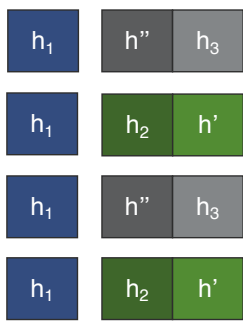

Figure 8: (a) Representation of the pentamer structure of $\mathbf{B 4} \mathbf{p}$. $\mathrm{h}_{1}$ bonds are also highlighted by the blue frames to illustrate the tilt of the amide moieties. Schematic representation of the H-Bond framework between the monomers with (b) the expected framework with perfectly stacked monomers and (c) the framework that is observed during the simulations with the formation and alternation of hybrid bonds.

For B4s family the conclusions are different with respect to B9s. Indeed, for the entire B4s family, the pentamers appeared as stable along the trajectory (Figure 8 for B4p and Figure S5, S6, and S7 in ESI $\dagger$ for B4, B4c, B4pI respectively). The $\mathrm{H}$-Bond network is at the origin of this stability. As for the dimers, $\mathrm{h}_{1}$ is always effective and can thus be considered as the backbone of the entire supramolecular assembly. Within the pentamer, amide moieties are no longer perpendicular to the plane of the molecules, leading on average to a tilt of $15^{\circ}(8(a))$. The fact that the amide moieties are quite flexible also allows the formation of the previously mentioned hybrid bonds. During the simulation, there is an alternation of $h_{2} / h$ ' and $h_{3} / h$ " bonds (Figure 8 (c)) which implies that the monomers are stacked in staggered rows. The interactions between each pair of monomers and the stability of this interaction is provided by the formation of one expected H-Bond $\left(h_{1}\right)$ and an hybrid scheme composed of two H-Bonds ( $h_{2} / h$ ' or $h_{3} / h$ "). This finding confirms the importance 
of the slight flexibility of the amide moieties for the global stability of SMA. It also highlights the fact that the stability of the SMA is dynamic as there is a constant compensation of the H-bonds.

\subsection{Aggregation mode}

Once the strength of the different supramolecular assemblies (dimers to pentamers) have been considered from both a qualitative and quantitative point of view, we decided to have a look at the formation of those assemblies. In the previous sections, we reported data about already formed supramolecular assemblies, in this section, we will study the aggregation and association process, aiming at answering the question: How do we go from monomers to larger assemblies? Different simulations with similar starting configurations were set up as follows to address different problems:

- Non-interacting monomers (no H-Bond between them) placed in the center of the simulation box for a long (100 ns) simulation time.

- Non-interacting monomers (no H-Bond between them) placed in the center of the simulation box for 100 short (1 ns) simulation times.

The first type of simulation will allow us to know if SMA can self-assemble spontaneously and if this SMA will be stable along a long simulation time. The second type of simulation will provide information on the frequency of formation of such SMA and more precisely the frequency of formation of H-Bonds. For simplicity, results and detailed analysis on B4p are presented here and in Supporting Information for the others.

\subsubsection{One long simulation}

We used PACKMOL[44] to generate a starting configuration encompassing 10 monomers that are loosely compacted, meaning they are relatively close to each other but with no hydrogen bonds or particular interaction between them. They were then placed in the center of a simulation box for a $100 \mathrm{~ns}$ long simulation time. To ensure that the monomers are not interacting at the beginning of the simulation and more particularly that no hydrogen bond is effective for the starting configuration, we represented the radial distribution function (RDF) for the oxygen and hydrogen atoms involved in those interactions (see Figure 9 (a)). The atoms $\mathrm{O}_{1}$, $\mathrm{O}_{2}$ and $\mathrm{O}_{3}$ and $\mathrm{H}_{1}, \mathrm{H}_{2}$ and $\mathrm{H}_{3}$ are the ones associated to $\mathrm{h}_{1}, \mathrm{~h}_{2}$ and $\mathrm{h}_{3}$ hydrogen bond identified on Figure 5. One can observe on Figure 9 (a) that for each oxygen atom $\left(\mathrm{O}_{1}, \mathrm{O}_{2}\right.$ and $\left.\mathrm{O}_{3}\right)$ there is no peak indicating a possible hydrogen bond with either $\mathrm{H}_{1}, \mathrm{H}_{2}$ or $\mathrm{H}_{3}$ for the starting configuration. The first and most intense peak corresponds to an intramolecular peak at around $3.0 \mathrm{~nm}$ corresponding to oxygen and hydrogen atoms of the same amide moiety. The following peaks, appearing at distances larger than $3.0 \mathrm{~nm}$, are indicating the relative spacing of the different $\mathrm{O}$ and $\mathrm{H}$ atoms and thus of the different monomers. We have then represented the same 
(a)
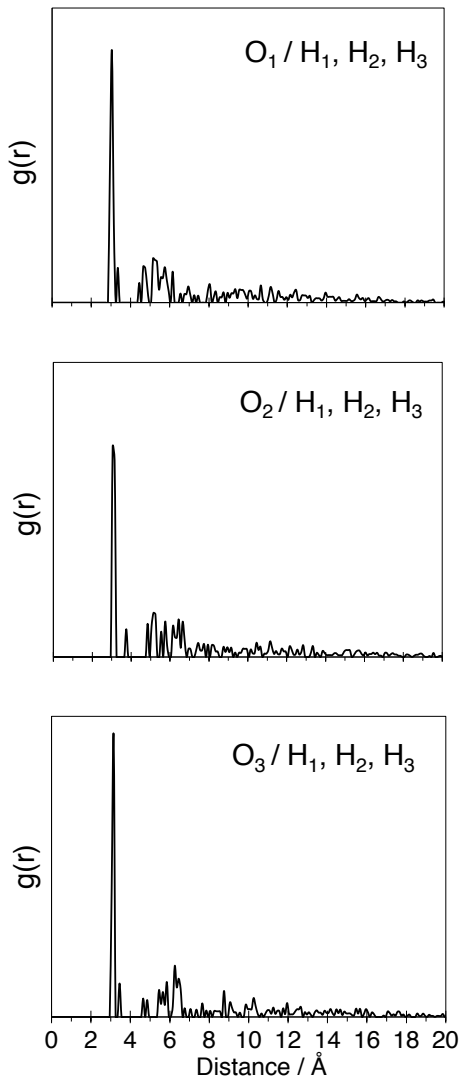

(b)
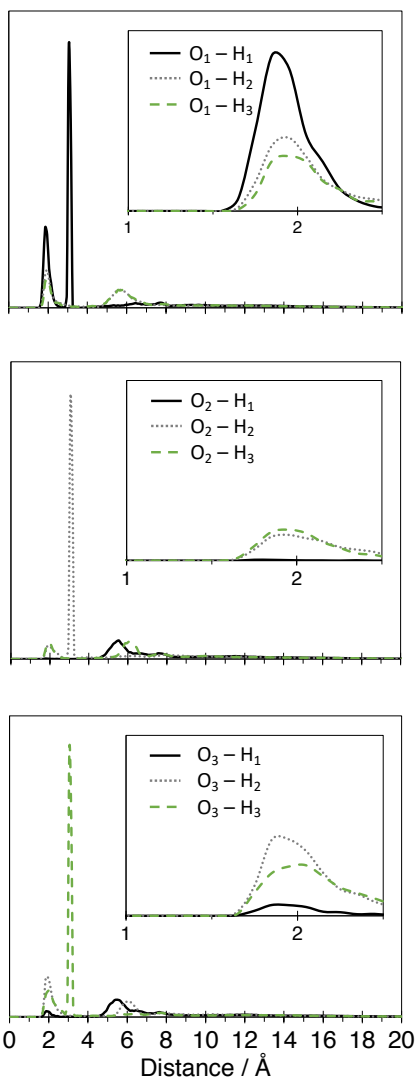

Figure 9: Radial distribution function for a B4p aggregate containing 10 monomers. (a) For the starting configuration ( $\mathrm{t}=0 \mathrm{~s})$ and (b) for a $100 \mathrm{~ns}$ long simulation. The attention has been focused on the oxygen and hydrogen atoms involved in possible H-Bond (see Figure 5 .

RDF but for a $100 \mathrm{~ns}$ long simulation, differentiating the three possible H-Bond that can be formed between two monomers (Figure 9 (b)). One can observe that the $\mathrm{O}_{1}-\mathrm{H}_{1}$ is predominantly formed when compared to possible $\mathrm{O}_{1}-\mathrm{H}_{2}$ and $\mathrm{O}_{1}-\mathrm{H}_{3} \mathrm{H}$-Bonds. Looking at the two other graphics, one can say that $\mathrm{O}_{2}$ is almost not implied in $\mathrm{H}$-Bond while some $\mathrm{O}_{3}-\mathrm{H}_{2}$ and $\mathrm{O}_{3}-\mathrm{H}_{3}$ are effective. If $\mathrm{O}_{3}-\mathrm{H}_{3}$ is an expected $\mathrm{H}$-Bond in the case of a perfectly aligned dimer, $\mathrm{O}_{3}-\mathrm{H}_{2} \mathrm{H}$-Bond corresponds to the hybrid $\mathrm{H}$-Bond already mentioned in the previous section, proving once again the importance of such interaction.

On Figure 10 we have provided a representative structure of a SMA of B4p, involving 8 monomers, that has been formed during the simulation. One has to notice that the two remaining monomers are involved in a dimer that is not interacting with the octamer. To illustrate the interactions between the different monomers we also provided interaction surfaces extracted from the NCI analysis for each pair of monomers. Various 

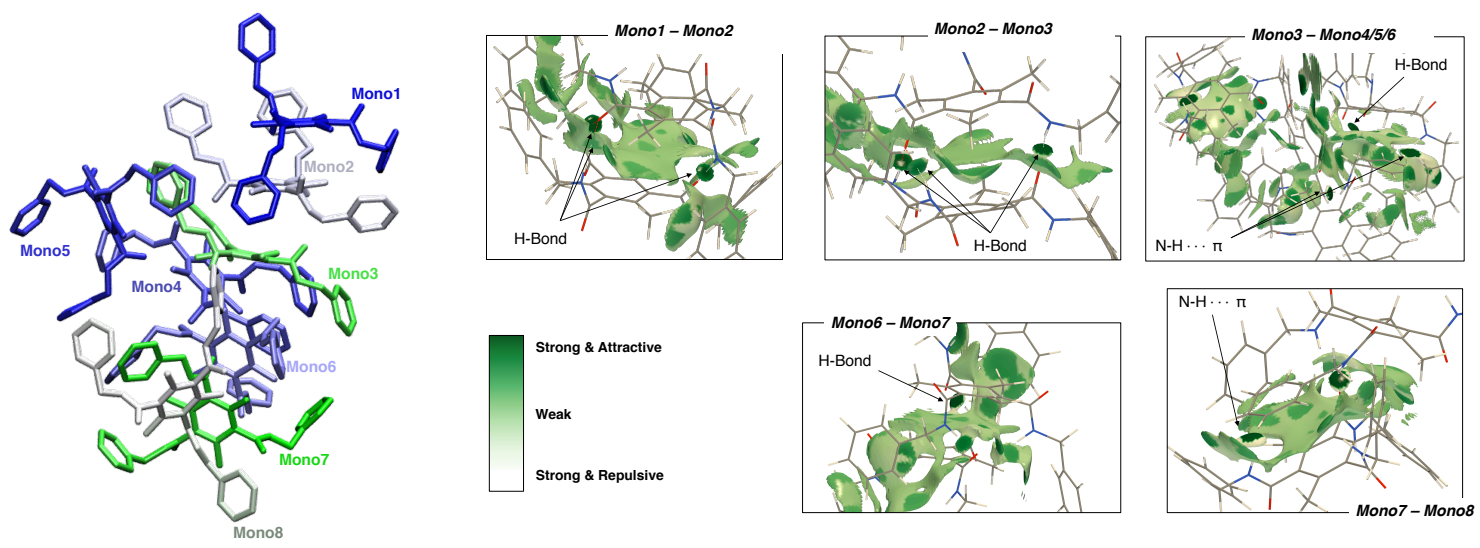

Figure 10: Representative structure of B4p aggregate extracted from the $100 \mathrm{~ns}$ long simulation along with the $\mathrm{NCI}$ analysis. Isosurfaces ( $s=0.5 \mathrm{a} . \mathrm{u}$ ) in dark green represent strong and attractive interactions (e.g H-Bonds), whereas weak interactions such as van der Waals interactions are depicted in light green. For the sake of clarity for the structure on the left, hydrogen atoms have not been represented, except those of the amide moieties.

interactions have been highlighted among which the predominant ones are:

- $\mathrm{h}_{1}$ H-Bond (Mono2-Mono3, Mono6-Mono7)

- $\mathrm{h}_{2}\left(\mathrm{~h}_{3}\right)$ associated with h' (h") hybrid H-Bond (Mono3-4/5/6)

- N-H $\cdots \pi$ bond (Mono3-Mono4/5/6, Mono7-Mono8)

- $\mathrm{H}$-Bond involving $\mathrm{O}_{1}$ atoms and $\mathrm{H}_{2} / \mathrm{H}_{3}$ atoms (Mono1-Mono2, Mono7-Mono8)

One has to notice that other interactions can be found within the SMA, for example $\pi$ - $\pi$ interactions between the phenyls of different monomers and $\mathrm{C}-\mathrm{H} \cdots \pi$ interactions. Those interactions are nevertheless more labile. If we go deeper in the analysis of the different interactions, it is possible to observe that all the interactions are not formed simultaneously but sequentially. Indeed the first interaction that is effective within the SMA is the $\mathrm{h}_{1}$ bond, with a formation at the nanosecond timescale (see Figure 11 . We then have the formation of the previously mentioned interactions ( $\mathrm{h}_{2}$ and $\mathrm{h}$ ') at a slightly higher timescale (few nanoseconds), followed by $\mathrm{N}-\mathrm{H} \cdots \pi$ interactions and $\mathrm{O}_{1}-\mathrm{H}_{2} / \mathrm{H}_{3}$ after tens of nanosecond. If $\mathrm{h}_{1}$ is the first interaction being observed, it is also the most stable one as it is effective during almost the entire trajectory (Figure 11.b)). For other molecules of the B4s family, there is always the formation of a SMA involving at least five monomers (B4 and B4pI). B4p, with 8 monomers involved is the most efficient one while B4c forms a seven-members SMA (see Figure S10, S11, and S12 in ESI $\dagger$ ). The same kind of interactions are present within all the SMA with a predominance for the $\mathrm{h}_{1}$ bond, completed with the previously mentioned interactions.

On the opposite, when considering B9 and B9m molecules, no large SMA were detected and only poorly stable dimers were observed. As a conclusion one can say that the self-assembling process is (1) efficient for B4s family but not for the B9s one, (2) quite fast, of the order of the nanosecond, (3) can be a long process as the interactions are added sequentially, and (4) dynamic in the sense that some interactions $\left(\mathrm{N}-\mathrm{H} \cdots \pi, \mathrm{O}_{1}-\mathrm{H}_{2}\right.$, 

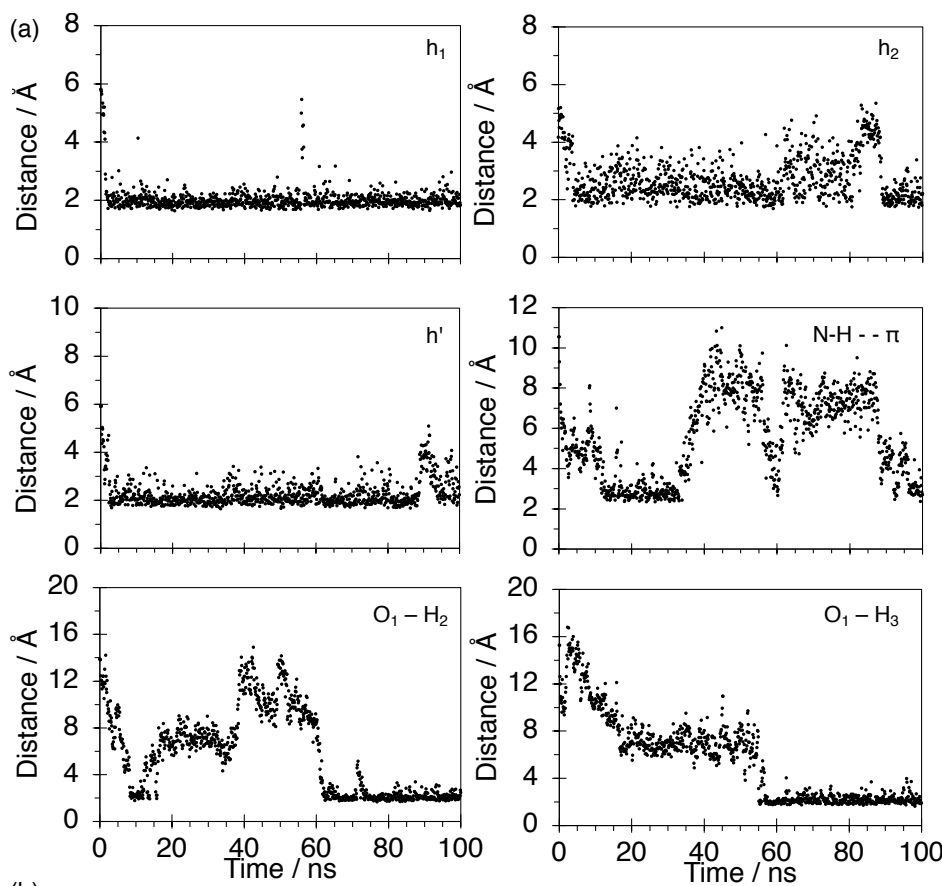

(b)

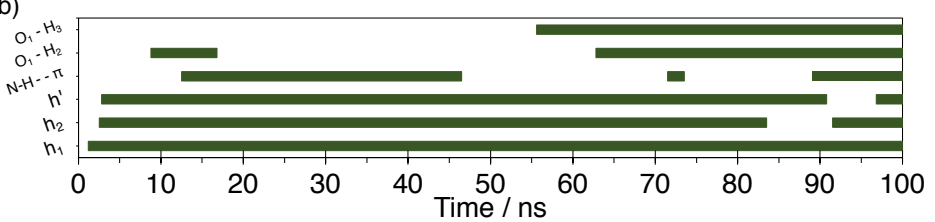

Figure 11: (a) Evolution for B4p octamer of the distances $h_{1}, h_{2}, h$ ', N-H $\cdots \pi, \mathrm{O}_{1}-\mathrm{H}_{2}, \mathrm{O}_{1}-\mathrm{H}_{3}$ during a $100 \mathrm{~ns}$ long simulation. All the distances are in $\AA$. (b) Schematic representation of the formation time and effectiveness of the previously mentioned interactions.

$\left.\mathrm{O}_{1}-\mathrm{H}_{3}\right)$ are labile but also compensated by other interactions $(\mathrm{C}-\mathrm{H} \cdots \pi, \pi-\pi)$, ensuring the global stability of the SMA.

\subsubsection{Many short simulations}

We observed in the previous section that, when a H-Bond is formed, it is then quite stable along the trajectory. The analysis we propose to perform in this section aims at retrieving the frequency of formation of the $\mathrm{H}-\mathrm{Bond}$. To do so, we generated 100 different starting configurations encompassing 10 monomers close enough to each other but with no H-Bond between them and performed a $1 \mathrm{~ns}$ simulation. We then extracted the final structure of each simulation and counted the H-Bonds between all the monomers. One has to notice that no distinction was made between all the possible H-Bonds (9 in total). On Figure 12 we have represented, for the 100 simulations and for B4p and B9m only, the total number of H-Bonds that have been observed between each pair of dimers. The results for B4, B4c, B4pI and B9 are also provided in ESI $\dagger$ (Figure S13 and S14). 


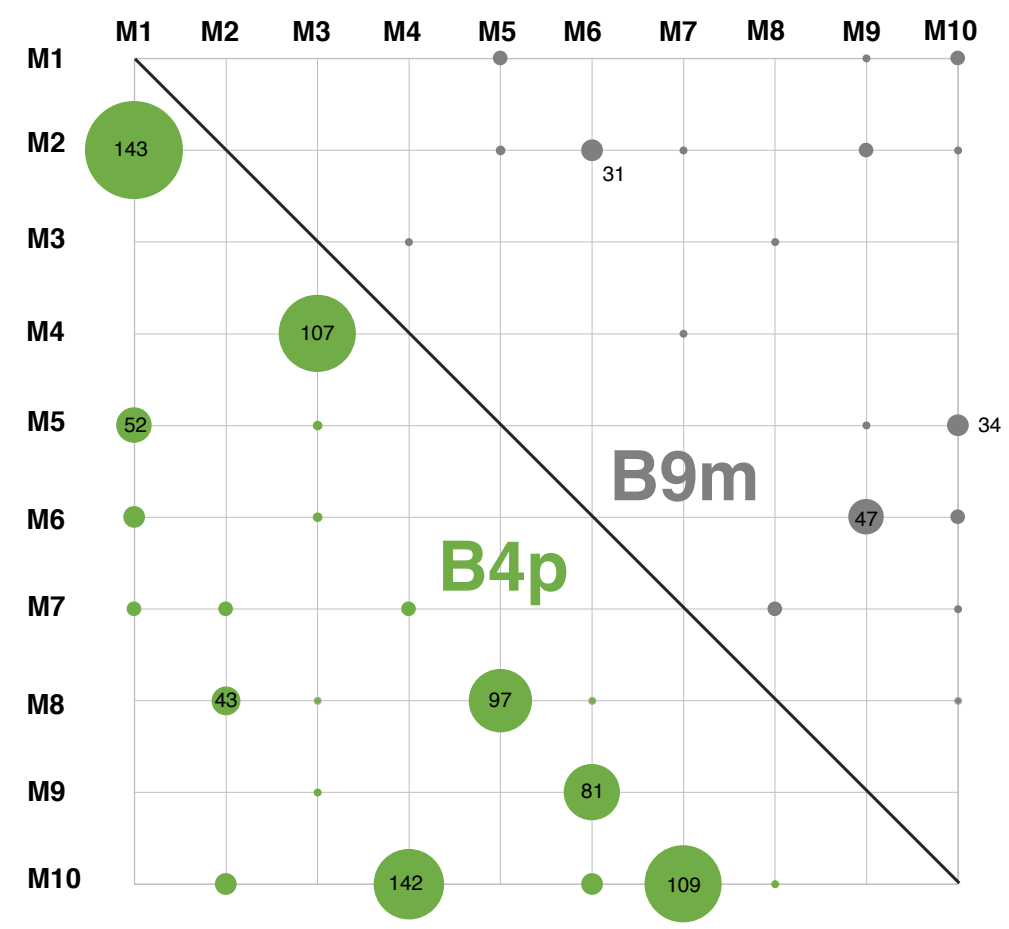

Figure 12: Schematic representation of the occurrence of the formation of H-Bonds between all the monomers (Mi with $\mathrm{i}=1, \ldots, 10$ ) for B4p (bottom left, green) and B9m (up right, gray) for a total of 100 simulations. The structures that are considered for the count are the final ones obtained at the end of the $1 \mathrm{~ns}$ simulation. The size of the dots is proportionnal to the number of H-Bonds that have been detected. For the largest dots, we provided the corresponding exact number of interactions.

One can observe immediately on Figure 12 that the number of H-Bonds present within B4p aggregate is larger than the ones of $\mathbf{B 9 m}$. Due to the particular arrangement of the molecules, some preferential interactions are observed. For example, for B4p, the formation of H-Bond between monomer 1 and monomer 2 is almost systematic. Indeed, the count reveals $143 \mathrm{H}$-bonds between those 2 monomers for a maximum of 300 . Nevertheless, one can also observe that monomer 1 is also involved in interactions with monomer 5 (52) and in a lesser extent with monomer 6 (31) and 7 (18). So does monomer 2 with monomer 7 (17), 8 (43) and 10 (29). For B4p, there are six main interactions involving M1-M2, M3-M4, M4-M10, M10-M7 M5-M8, and M6-M9. For information, all the monomers are staying close to each other, as a loose aggregate, during all the simulations. This can be explained by the fact that other interactions $(\mathrm{C}-\mathrm{H} \cdots \pi$ and N-H $\cdots \pi)$ are formed and thus enforce the stability of the entire supramolecular assembly. For B9m, no systematic interaction was observed with a maximum count observed for a M6-M9 interaction. This result may also be due to the fact that B9m monomers are not staying "packed" during the simulation. The loose aggregate is thus not even stable for B9m molecules. 


\subsection{Conclusion}

By defining a complete theoretical protocol based mainly on molecular dynamics simulations and aiming at studying the ability of organic molecules to form supramolecular assemblies and their resulting stabilities, we have been able to provide some hints for an effective self-supramolecular assembling process. A total of 6 molecules (B4, B4c, B4p, B4pI, B9, and B9m) divided into 2 families (B4s and B9s), bearing a different backbone but with three amide moieties in common have been considered. The study of the monomers allowed us to validate our molecular dynamics approach and also to understand the properties of the molecules when they are isolated. Considering the dimers has permitted to define the hydrogen bonds network. Expected hydrogen bonds $\left(h_{1}, h_{2}\right.$, and $\left.h_{3}\right)$ were always observed for both families. Nevertheless, they were not always effective all together and an hybrid scheme (h' and h") has been highlighted. This hybrid H-bond network was observed only for one of the two families, namely B4s. The calculation of the binding energies clearly showed that dimers and even trimers or pentamers of B4s family were more favorable than those of the B9s. Nevertheless, the evolution of the binding energy going from dimers to pentamers indicates that small oligomers (e.g trimers) may be more stabilized than larger SMA (e.g pentamers). Looking at the the formation of the assemblies starting from a loose aggregate allowed us to observe that not only H-bonds can ensure the stability of the aggregate but also $\mathrm{N}-\mathrm{H} \cdots \pi$ or $\mathrm{C}-\mathrm{H} \cdots \pi$ in a lesser extent. Finally, the most important point to consider for the self-assembling process is the dynamical behavior of the stability. If SMA of B4s family are more stable than those of B9s it is because they have a relative flexibility. One can also notice that the stability is not something that is frozen but that is also dynamical. We have shown within this study that considering relatively flexible molecules, instead of rigid ones, is a better strategy for the conception of supramolecular assemblies.

\section{Supporting Information}

See supporting information for: Validation of GAFF parameters; General parameters for molecular dynamics simulations; Parameters for the umbrella sampling approach; Particular structure of B4s and B9s families; Hybrid hydrogen bond network; Pentamers for B4, B4c, B4pI, B9, and B9m; Interaction maps for B4, B4c, B4pI and B9.

\section{Acknowledgements}

The authors thank Dr J. Quertinmont, C. Bouquiaux, P. Beaujean and C. D. Matatu MBengo for fruitful scientific discussions. This work was carried out thanks to fundings from the Fonds de la Recherche du Québec (F.R.Q.) et du Fonds de la Recherche Scientifique (F.R.S.-FNRS) in the frame of bilateral research 
projects under convention R.P001.19. L.L.B. thanks the F.R.S.-FNRS for her post-doctoral grant (convention R.P001.19). The calculations were performed on the computers of the «Consortium des équipements de Calcul Intensif (CÉCI) » (http://www.ceci-hpc.be), including those of the «UNamur Technological Platform of High-Performance Computing (PTCI)» (http://www.ptci.unamur.be), for which we gratefully acknowledge the financial support from the FNRS-FRFC, the Walloon Region, and the University of Namur (Conventions No. 2.5020.11, GEQ U.G006.15, U.G018.19, 1610468, and RW/GEQ2016) as well as on Zenobe, the Tier-1 facility of the Walloon Region (Convention 1117545).

\section{References}

[1] P. J. Cragg. Supramolecular Chemistry and the Life Sciences, pages 49-89. Springer Netherlands, Dordrecht, 2010.

[2] J. D. Hartgerink, E. Beniash, and S. I. Stupp. Self-assembly and mineralization of peptide-amphiphile nanofibers. Science, 294(5547):1684-1688, 2001.

[3] N. Kameta, M. Masuda, G. Mizuno, N. Morii, and T. Shimizu. Supramolecular nanotube endo sensing for a guest protein. Small, 4(5):561-565, 2008.

[4] J. T. Ernst, O. Kutzki, A. K. Debnath, S. Jiang, H. Lu, and A. D. Hamilton. Design of a protein surface antagonist based on $\alpha$-helix mimicry: Inhibition of gp41 assembly and viral fusion. Angew. Chem. Int. Ed, 41(2):278-281, 2002.

[5] D. H. Williams and B. Bardsley. The vancomycin group of antibiotics and the fight against resistant bacteria. Angew. Chem. Int. Ed, 38(9):1172-1193, 1999.

[6] Y. Q. Wen, Y. L. Song, G. Y. Jiang, D. B. Zhao, K. Ding, W. F. Yuan, X. Lin, H. J. Gao, L. Jiang, and D. B. Zhu. Crystalline thin films formed by supramolecular assembly for ultrahigh-density data storage. Adv. Mater., 16(22):2018-2021, 2004.

[7] J.-M. Lehn. Supramolecular Chemistry. John Wiley \& Sons, Ltd, 1995.

[8] P. J. Cragg. An Introduction to Supramolecular Chemistry, pages 1-48. Springer Netherlands, Dordrecht, 2010.

[9] C. W. Bunn, E. V. Garner, and W. L. Bragg. The crystal structures of two polyamides ("nylons"). Proc. R. Soc. Lond. A, 189(1016):39-68, 1947.

[10] A. V. Morozov and T. Kortemme. Potential functions for hydrogen bonds in protein structure prediction and design. In Peptide Solvation and HBonds, volume 72 of Advances in Protein Chemistry, pages 1-38. Academic Press, 2005. 
[11] E. T. Powers, S. Deechongkit, and J. W. Kelly. Backbone-backbone h-bonds make context-dependent contributions to protein folding kinetics and thermodynamics: Lessons from amide-to-ester mutations. In Peptide Solvation and HBonds, volume 72 of Advances in Protein Chemistry, pages 39-78. Academic Press, 2005.

[12] A. Greenberg, C. Breneman, and J. F. Liebman. In The amide linkage : structural significance in chemistry, biochemistry, and materials science, 2003.

[13] Y. Matsunaga, N. Miyajima, Y. Nakayasu, S. Sakai, and M. Yonenaga. Design of novel mesomorphic compounds: N,N',N"-trialkyl-1,3,5-benzenetricarboxamides. Bull. Chem. Soc. Jap., 61(1):207-210, 1988.

[14] Y. Shishido, H. Anetai, T. Takeda, N. Hoshino, S.-i. Noro, T. Nakamura, and T. Akutagawa. Molecular assembly and ferroelectric response of benzenecarboxamides bearing multiple $-\mathrm{CONHC}_{14} \mathrm{H}_{29}$ chains. $J$. Phys. Chem. C, 118(36):21204-21214, 2014.

[15] L. Brunsveld, A.P.H.J. Schenning, M.A.C. Broeren, H.M. Janssen, J.A.J.M. Vekemans, and E.W. Meijer. Chiral amplification in columns of self-assembled N,N',N'"-tris((s)-3,7-dimethyloctyl)benzene-1,3,5tricarboxamide in dilute solution. Chem. Lett., 29(3):292-293, 2000.

[16] J. Roosma, T. Mes, P. Leclère, A. R. A. Palmans, and E. W. Meijer. Supramolecular materials from benzene-1,3,5-tricarboxamide-based nanorods. J. Am. Chem. Soc., 130(4):1120-1121, 2008.

[17] P. J. M. Stals, M. M. J. Smulders, R. Martín-Rapún, A. R. A. Palmans, and E. W. Meijer. Asymmetrically substituted benzene-1,3,5-tricarboxamides: Self-assembly and odd-even effects in the solid state and in dilute solution. Chem. Eur. J., 15(9):2071-2080, 2009.

[18] P. J. M. Stals, P. A. Korevaar, M. A. J. Gillissen, T. F. A. de Greef, C. F. C. Fitié, R. P. Sijbesma, A. R. A. Palmans, and E. W. Meijer. Symmetry breaking in the self-assembly of partially fluorinated benzene-1,3,5-tricarboxamides. Angew. Chem. Int. Ed., 51(45):11297-11301, 2012.

[19] L. Le Bras, R. Berthin, M. Hamdi, I.and Louati, S. Aloïse, M. Takeshita, C. Adamo, and A. Perrier. Understanding the properties of dithienylethenes functionalized for supramolecular self-assembly: a molecular modeling study. Phys. Chem. Chem. Phys., 22:6942-6952, 2020.

[20] J. A. Lemkul and D. R. Bevan. Assessing the stability of alzheimer's amyloid protofibrils using molecular dynamics. J. Phys. Chem. B, 114(4):1652-1660, 2010.

[21] F. Mahmoudinobar, J. M. Urban, Z. Su, B. L. Nilsson, and C. L. Dias. Thermodynamic stability of polar and nonpolar amyloid fibrils. J. Chem. Theory Comput., 15(6):3868-3874, 2019.

[22] I. M. Ilie and A. Caflisch. Simulation studies of amyloidogenic polypeptides and their aggregates. Chem. Rev., 119(12):6956-6993, 2019. 
[23] N. T. Tung, P. Derreumaux, V. V. Vu, P. C. Nam, and S. T. Ngo. C-terminal plays as the possible nucleation of the self-aggregation of the s-shape $\mathrm{A} \beta_{11-42}$ tetramer in solution: Intensive md study. ACS Omega, 4(6):11066-11073, 2019.

[24] E. R. Johnson, S. Keinan, P. Mori-Sánchez, J. Contreras-García, A. J. Cohen, and W. Yang. Revealing noncovalent interactions. J. Am. Chem. Soc., 132(18):6498-6506, 2010.

[25] A. Perrier, M. Eluard, M. Petitjean, and A. Vanet. In silico design of new inhibitors against hemagglutinin of influenza. J. Phys. Chem. B, 123(3):582-592, 2019.

[26] M. J. Frisch, G. W. Trucks, H. B. Schlegel, G. E. Scuseria, M. A. Robb, J. R. Cheeseman, G. Scalmani, V. Barone, G. A. Petersson, H. Nakatsuji, X. Li, M. Caricato, A. V. Marenich, J. Bloino, B. G. Janesko, R. Gomperts, B. Mennucci, H. P. Hratchian, J. V. Ortiz, A. F. Izmaylov, J. L. Sonnenberg, D. WilliamsYoung, F. Ding, F. Lipparini, F. Egidi, J. Goings, B. Peng, A. Petrone, T. Henderson, D. Ranasinghe, V. G. Zakrzewski, J. Gao, N. Rega, G. Zheng, W. Liang, M. Hada, M. Ehara, K. Toyota, R. Fukuda, J. Hasegawa, M. Ishida, T. Nakajima, Y. Honda, O. Kitao, H. Nakai, T. Vreven, K. Throssell, J. A. Montgomery, Jr., J. E. Peralta, F. Ogliaro, M. J. Bearpark, J. J. Heyd, E. N. Brothers, K. N. Kudin, V. N. Staroverov, T. A. Keith, R. Kobayashi, J. Normand, K. Raghavachari, A. P. Rendell, J. C. Burant, S. S. Iyengar, J. Tomasi, M. Cossi, J. M. Millam, M. Klene, C. Adamo, R. Cammi, J. W. Ochterski, R. L. Martin, K. Morokuma, O. Farkas, J. B. Foresman, and D. J. Fox. Gaussian 16 Revision C.01, 2016. Gaussian Inc. Wallingford CT.

[27] J.-D. Chai and M. Head-Gordon. Long-range corrected hybrid density functionals with damped atomatom dispersion corrections. Phys. Chem. Chem. Phys., 10:6615-6620, 2008.

[28] K. S. Thanthiriwatte, E. G. Hohenstein, L. A. Burns, and C. D. Sherrill. Assessment of the performance of DFT and DFT-D methods for describing distance dependence of hydrogen-bonded interactions. $J$. Chem. Theory Comput., 7(1):88-96, 2011.

[29] J. Tomasi, B. Mennucci, and R. Cammi. Quantum mechanical continuum solvation models. Chem. Rev., 105(8):2999-3094, 2005.

[30] H. P. Hratchian, A. V. Krukau, P. V. Parandekar, M. J. Frisch, and K. Raghavachari. QM:QM embedding using electronic densities within an ONIOM framework: Energies and analytic gradients. J. Chem. Phys., 135(1):014105, 2011.

[31] J. Contreras-García, E. R. Johnson, S. Keinan, R. Chaudret, J.-P. Piquemal, D. N. Beratan, and W. Yang. NCIPLOT: A program for plotting noncovalent interaction regions. J. Chem. Theory Comput., 7(3):625$632,2011$. 
[32] J. Wang, R. M. Wolf, J. W. Caldwell, P. A. Kollman, and D. A. Case. Development and testing of a general amber force field. J. Comput. Chem., 25:1157-1174, 2004.

[33] M. J. Abraham, T. Murtola, R. Schulz, S. Páll, J. C. Smith, B. Hess, and E. Lindahl. GROMACS: High performance molecular simulations through multi-level parallelism from laptops to supercomputers. SoftwareX, 1-2:19-25, 2015.

[34] J. Wang, R. M. Wolf, J. W. Caldwell, P. A. Kollman, and D. A. Case. Development and testing of a general amber force field. J. Comput. Chem., 25(9):1157-1174, 2004.

[35] J. Wang, W. Wang, P. A. Kollman, and D. A. Case. Automatic atom type and bond type perception in molecular mechanical calculations. J. Mol. Graph., 25(2):247 - 260, 2006.

[36] P. R. Batista, A. Wilter, E. H. A. B. Durham, and P. G. Pascutti. Molecular dynamics simulations applied to the study of subtypes of HIV-1 protease common to Brazil, Africa, and Asia. Cell Biochem. Biophys., 44(3):395-404, Mar 2006.

[37] T. Darden, D. York, and L. Pedersen. Particle mesh ewald: An N $\log (\mathrm{N})$ method for ewald sums in large systems. J. Chem. Phys., 98(12):10089-10092, 1993.

[38] U. Essmann, L. Perera, M. L. Berkowitz, T. Darden, H. Lee, and L. G. Pedersen. A smooth particle mesh ewald method. J. Chem. Phys., 103(19):8577-8593, 1995.

[39] H. J. C. Berendsen, J. P. M. Postma, W. F. van Gunsteren, A. DiNola, and J. R. Haak. Molecular dynamics with coupling to an external bath. J. Chem. Phys., 81(8):3684-3690, 1984.

[40] J. S. Hub, B. L. de Groot, and D. van der Spoel. g_wham - a free weighted histogram analysis implementation including robust error and autocorrelation estimates. J. Chem. Theory Comput., 6(12):3713-3720, 2010.

[41] C. H. Görbitz. Peptide structures. Current Opinion in Solid State and Materials Science, 6(2):109-116, 2002.

[42] P. Baillargeon, S. Bernard, D. Gauthier, R. Skouta, and Y. L. Dory. Efficient synthesis and astonishing supramolecular architectures of several symmetric macrolactams. Chem. Eur. J., 13(33):9223-9235, 2007.

[43] Thomas Marmin and Y. L. Dory. Self-assembly of $\mathrm{C}_{3}$ symmetric rigid macrolactams into very polar and porous trigonal crystals. Chem. Eur. J., 25(27):6707-6711, 2019.

[44] L. Martínez, R. Andrade, E. G. Birgin, and J. M. Martínez. Packmol: A package for building initial configurations for molecular dynamics simulations. J. Comput. Chem., 30(13):2157-2164, 2009. 